LPTHE Orsay $98 / 64$

November 1998

\title{
THE LIKELIHOOD OF DCC FORMATION
}

\author{
André KRZYWICKI and Julien SERREAU \\ LPTHE, Bâtiment 211, Université Paris-Sud, 91405 Orsay, France'
}

\begin{abstract}
We estimate the probability that a disoriented chiral condensate forms during the spherical expansion of a hot medium described by the linear sigma model.
\end{abstract}

\section{Introduction}

The suggestion [1]- [4] that disoriented chiral condensates (DCC) could form in high-energy hadronic collisions has attracted much attention. More than hundred publications are devoted to the study of various aspects of this hypothetical phenomenon (see e.g. the reviews [0, 6]). The most plausible mechanism of DCC formation has been identified: fast expansion of hot quark-gluon plasma results in a rapid suppression of thermal fluctuations (quenching), which in turn triggers a dramatic amplification of soft pion modes (see e.g. refs. [7, 8, 9]. However, nobody made a serious attempt to estimate the probability that this happens. It is true that the present models are not realistic enough to be trusted at the quantitative level. Nevertheless, even within the existing framework it is legitimate to seek for a crude estimate of the probability in question. This is the problem we address in the present paper.

It is expected that the chiral symmetry is restored in a sufficiently hot quark-gluon plasma and that it is spontaneously broken when the plasma is cooled. At the same time, freely propagating quarks and gluons get trapped into hadrons. It would be too difficult to model both phenomena, symmetry breaking and confinement. Hence, in the DCC literature it is customary to describe the soft modes of the cooling medium by the linear sigma model, evacuating cavalierly the confinement problem. The justification of this idealization, which we also adopt, is discussed at length in many places.

\footnotetext{
${ }^{1}$ Laboratoire associé au Centre National de la Recherche Scientifique - URA00063.
} 
The fast expansion can be modeled by assuming that the field depends on time via the variable $\tau=\sqrt{t^{2}-x^{\mu} x_{\mu}}$, the index $\mu$ running from 1 to $D$. In the equations of motion the operator $\partial^{2} / \partial t^{2}$ is then replaced by $\partial^{2} / \partial \tau^{2}+$ $(D / \tau) \partial / \partial \tau$. In addition to the acceleration term, there also appears a "friction" term reflecting the decrease of energy in a covolume [3]. Since the "friction force" is proportional to $D$, the larger is $D$ the more efficient is the quenching [9].

In this paper we focus on the spherical geometry, the most favourable one for DCC formation. We imagine a droplet of DCC undergoing a radial expansion in its rest frame. As remarked in [10], where the same geometry is studied, this does not necessarily mean that the full collision process has the same symmetry.

The time evolution of the chiral phase transition during a spherical expansion of a medium described by the linear sigma model was studied in ref. [8]. We adopt most of their formalism here and, in particular, the two basic ingredients: the assumption that the system is initially in local thermal equilibrium and the self-consistent approximation of the mean-field type. We shall argue that essentially no other assumptions are needed to determine the probability that soft modes are amplified by a given amount. This is, however, not quite enough to fix the probability of observing a DCC in a collision process. Information on the environement of the DCC bubble and on the experimental set-up is needed for that. We shall come back to this question towards the end of this paper.

In the next section we review briefly the formalism. In sect. 3 we explain our sampling strategy for initial conditions. The results and discussion are in sect. 4 .

\section{The formalism}

Let us briefly summarize the formalism of ref. [8]. The authors start with the linear sigma model for an $N$-component scalar field (eventually $N$ is set to 4 ). Using the $1 / N$ approximation they derive the following dynamical equations of the Hartree type:

$$
\begin{aligned}
& \left(\partial^{2}+\chi\right) \overline{\boldsymbol{\phi}}(x)=H \mathbf{n}_{\sigma}, \\
& \left(\partial^{2}+\chi\right) \boldsymbol{\phi}(x)=0
\end{aligned}
$$

where $\chi$ satisfies the gap equation

$$
\chi=\lambda_{0}\left(\bar{\phi}^{2}+\left\langle\phi^{2}\right\rangle-v_{0}^{2}\right),
$$

$\bar{\phi}$ is the classical mean-field and $\phi$ is the quantum fluctuation. The parameters $\lambda_{0}$ and $v_{0}$ are the bare couplings of the sigma model. An explicit symmetry 
breaking term, proportional to $H$, gives a finite mass to the Goldstone boson. Of course, $\left\langle\phi^{2}\right\rangle$ diverges and it is necessary to set an ultra-violet cut-off $\Lambda$. The quadratic divergence is removed by subtracting the mass gap at zero temperature, $T=0$, obtaining

$$
\frac{1}{\lambda_{0}}\left(\chi-m_{\pi}^{2}\right)=\bar{\phi}^{2}-f_{\pi}^{2}+\left\langle\phi^{2}\right\rangle-\left\langle\phi^{2}\right\rangle_{T=0}
$$

The remaining logarithmic divergence is eliminated by introducing the renormalized coupling constant:

$$
\lambda_{r}^{-1}=\lambda_{0}^{-1}+\left(N / 8 \pi^{2}\right) \int_{0}^{\Lambda} k^{2} d k /\left(k^{2}+m_{\pi}^{2}\right)^{3 / 2}
$$

The cancellation of divergences is, at any time, insured by an appropriate quantization condition (cf eqs. (111) below). The theory becomes trivial when $\Lambda \rightarrow \infty$ and therefore the cut-off has to be kept finite. The effect of the renormalization is to reduce the cut-off dependence of the results.

Notice, that in this approximation, unlike in the standard Hartree one, all components of the fluctuation field have the same mass $\sqrt{\chi}$, whose value is independent of the orientation of the order parameter $\bar{\phi}$. This is not very realistic but is acceptable if one wishes to estimate only the amplification of the length of the field vector, without paying attention to its orientation in the internal space.

In order to take care of the expansion, the time $t$ and the radial coordinate $r$ are replaced by the hyperbolic coordinates

$$
\tau=\sqrt{t^{2}-r^{2}}, \quad \eta=\tanh ^{-1}(r / t)
$$

and the theory is quantized on hypersurfaces $\tau=$ const. It is assumed that the mean-field depends on $\tau$ only. Projecting the fluctuation field on the orthonormal eigenmodes $\mathcal{Y}_{\mathbf{s}}(\eta, \theta, \varphi)$ of the curved Laplacian (cf [11]; we use the shorthand notation $\mathbf{s}=(s, l, m), s$ is dimensionless ) one gets

$$
\begin{aligned}
\phi_{\mathbf{S}}(\tau) & =\psi_{s}(\tau) \mathbf{a}_{\mathbf{S}}+(-1)^{m} \bar{\psi}_{s}(\tau) \mathbf{a}_{-\mathbf{s}}^{\dagger} \\
\dot{\phi}_{\mathbf{S}}(\tau) & =\dot{\psi}_{s}(\tau) \mathbf{a}_{\mathbf{S}}+(-1)^{m} \overline{\bar{\psi}}_{s}(\tau) \mathbf{a}_{-\mathbf{S}}^{\dagger}
\end{aligned}
$$

where

$$
\left[a_{i \mathbf{s}}, a_{\mathrm{j} \mathbf{s}}^{\dagger},\right]=\delta_{i j} \delta_{\mathbf{s s}}
$$

and the dot represents the differentiation with respect to $\tau$. The problem can be reduced to the familiar one of a set of parametrically excited harmonic oscillators. For that purpose one replaces $\psi_{s} \rightarrow g_{s} / \tau$ and one introduces the "time" variable $u=\log \left(m_{\pi} \tau\right)$ to get

$$
\left[\frac{d^{2}}{d u^{2}}+\omega_{s}(u)\right] g_{s}(u)=0
$$


where $\omega_{s}(u)=\sqrt{s^{2}+\chi(\tau) \tau^{2}}$ is the dimensionless "frequency". The oscillators are coupled through the common mass. The physical frequency is $\omega_{s}(u) / \tau$.

The quantization is completed by choosing appropriate initial conditions for the mode functions. The cancellation of divergences required by the renormalization of the mass gap is insured if one adopts the following adiabatic condition'2 at $u=u_{0}$ :

$$
g_{s}\left(u_{0}\right)=g_{s}^{(0)}\left(u_{0}\right), \quad g_{s}^{\prime}\left(u_{0}\right)=g_{s}^{(1)}\left(u_{0}\right)
$$

with

$$
\begin{aligned}
& g_{s}^{(0)}(u)=1 / \sqrt{2 \omega_{s}(u)} \\
& g_{s}^{(1)}(u)=-\left[\omega_{s}^{\prime}(u) / 2 \omega_{s}(u)+i \omega_{s}(u)\right] g_{s}^{(0)}(u)
\end{aligned}
$$

the prime representing the differentiation with respect to $u$.

Assume that Heisenberg and Schroedinger representations coincide at $\tau=\tau_{0}$. One can then regard $a_{\mathbf{S}}^{\dagger}$ as the Schroedinger representation operator creating a particle with frequency $\omega_{s}\left(u_{0}\right) / \tau_{0}$. These are the particles appropriate for the description of the initial state. One can introduce analogously the particles with frequencies $\omega_{s}\left(u_{f}\right) / \tau_{f}$ appropriate to the final state and counted at $\tau=\tau_{f}$. Let $b_{\mathbf{S}}^{\dagger}$ denote the corresponding creation operator, in the Schroedinger representation. The Heisenberg representation field operators, given by (7)-(8), can then also be written

$$
\begin{aligned}
& \phi_{\mathbf{S}}(\tau)=\psi_{s}^{(0)}\left(\tau_{f}\right) \mathbf{b}_{\mathbf{S}}(\tau)+(-1)^{m} \bar{\psi}_{s}^{(0)}\left(\tau_{f}\right) \mathbf{b}_{-\mathbf{S}}^{\dagger}(\tau) \\
& \dot{\phi}_{\mathbf{S}}(\tau)=\psi_{s}^{(1)}\left(\tau_{f}\right) \mathbf{b}_{\mathbf{S}}(\tau)+(-1)^{m} \bar{\psi}_{s}^{(1)}\left(\tau_{f}\right) \mathbf{b}_{-\mathbf{S}}^{\dagger}(\tau)
\end{aligned}
$$

where $\psi_{s}^{(0)}(\tau)=g_{s}^{(0)}(u) / \tau$ and $\psi_{s}^{(1)}(\tau)=\left[g_{s}^{(1)}(u)-g_{s}^{(0)}(u)\right] / \tau^{2}$ while $\mathbf{b}_{\mathbf{S}}(\tau)=$ $U\left(\tau, \tau_{0}\right) \mathbf{b}_{\mathbf{s}} U^{\dagger}\left(\tau, \tau_{0}\right), U$ being the unitary operator connecting the two representations (when the potential is quadratic $U$ can be explicitly constructed 13], but we do not need this construction here 3 ). Using (7)-(8) and (14)-(15) one easily finds the Bogoliubov transformation connecting the operators $\mathbf{a}_{\mathbf{S}}, \mathbf{a}_{\mathbf{S}}^{\dagger}$ and $\mathbf{b}_{\mathbf{S}}(\tau), \mathbf{b}_{\mathbf{S}}^{\dagger}(\tau)$ :

$$
\mathbf{b}_{\mathbf{S}}(\tau)=\alpha(\tau) \mathbf{a}_{\mathbf{S}}+\beta(\tau)(-1)^{m} \mathbf{a}_{-\mathbf{s}}^{\dagger}
$$

where

$$
\begin{aligned}
\alpha_{s}(\tau) & =i\left[g_{s}^{\prime}(u) \bar{g}_{s}^{(0)}\left(u_{f}\right)-g_{s}(u) \bar{g}_{s}^{(1)}\left(u_{f}\right)\right] \\
\beta_{s}(\tau) & =i\left[\bar{g}_{s}^{\prime}(u) \bar{g}_{s}^{(0)}\left(u_{f}\right)-\bar{g}_{s}(u) \bar{g}_{s}^{(1)}\left(u_{f}\right)\right]
\end{aligned}
$$

\footnotetext{
${ }^{2}$ Only second order adiabatic condition is imposed. This may be insufficient in the general context 12] but is enough when one only seeks to insure the proper renormalization of the mass gap.

${ }^{3}$ The operator $U$ combines free propagation and squeezing. The latter takes care of the creation of particle pairs.
} 
Assuming

$$
\left\langle a_{i \mathbf{S}}^{\dagger} a_{j \mathbf{s}},\right\rangle=n_{i s}^{(a)}\left(\tau_{0}\right) \delta_{i j} \delta_{\mathbf{s s}}, \quad, \quad\left\langle a_{i \mathbf{s}} a_{j \mathbf{s}}{ }\right\rangle=0
$$

one finally obtains time $\tau$ for a given number of a-particles at time $\tau_{0}$ ] :

$$
n_{i s}^{(b)}(\tau)+\frac{1}{2}=A_{s}(\tau)\left[n_{i s}^{(a)}\left(\tau_{0}\right)+\frac{1}{2}\right]
$$

where

$$
A_{s}=2\left|\beta_{s}\right|^{2}+1
$$

The multiplicity $n_{s}^{(b)}(\tau)$ calculated at $\tau=\tau_{f}$ depends weakly on the choice of the reference final time $\tau_{f}$, when the latter is large. Assuming local thermal equilibrium in the initial state one sets $n_{s}^{(a)}\left(\tau_{0}\right)=\left\{\exp \left[\omega_{s}\left(u_{0}\right) / \tau_{0} T\right]-1\right\}^{-1}$.

\section{$3 \quad$ Physical picture and the sampling strategy}

As already mentioned in the Introduction, we consider the evolution of a spherical droplet of DCC in its rest frame. We start with a small ball of radius $R$, filled with hot matter in local thermal equilibrium. We assume that the ball expands at the speed of light. Due to the time dilation the temperature and the value of the mean-field stay approximately constant within a layer near the boundary of the ball. Of course, the width of this layer shrinks to zero with increasing time (this width equals the distance between the surface $\tau=R$ and the light cone). The equations of motion of the sigma model are supposed to describe what happens in the interior of the ball, the cooling observed as one moves away from the surface towards the center. We make first the unrealistic assumption that the DCC is connected forever to a heat bath kept at constant temperature, so that the process never stops. Later on we shall discuss the effect of switching the heat bath off.

The evolution of the droplet depends on the initial conditions set at $\tau=\tau_{0}=R$. It is easy to convince oneself that in the Hartree approximation the only relevant initial conditions are those concerning the mean-field and its time derivative (the fluctuations of the initial occupancy numbers appear

\footnotetext{
${ }^{4}$ The derivation given here differs from that of ref. [8], where the so-called adiabatic basis is unnecessarily used. Strictly speaking, the Bogoliubov transformation given by their eqs. (3.19) is not unitary when the frequency is imaginary in some time interval, since the exponential factor entering the definition of the adiabatic basis is then no longer a pure phase. We understand that in actual calculations the amplification factor was obtained from the formula derived for real frequencies. We reproduce their results using eqs. (17)-(18).

${ }^{5} \mathrm{~J}$. Randrup has emphasized that the amplification of vacuum fluctuations contributes significantly to the effect, which is therefore likely to be underestimated using classical equations of motion [14]
} 
only in the formula for the initial mass gap and approximately average to zero there). How to choose these parameters? In all publications up to now they were given values ad hoc. We shall argue, that the probability that $\bar{\phi}\left(\tau_{0}\right)$ and $\dot{\bar{\phi}}\left(\tau_{0}\right)$ take a given value is determined once one has assumed that the droplet is initially in local thermal equilibrium. In order to make the point let us for a moment neglect the complications due to quantum mechanics and let us us consider a classical field.

Consider a ball of fixed volume $V_{0}$ filled with the field in local thermal equilibrium. Hence, the field fluctuates as if the ball were part of a system in equilibrium, with volume $V$ much larger than $V_{0}$. In this large system the variances of the spacial averages of the field and of its time derivative would be very small, of order $1 / V$ (since long range correlations are absent). The corresponding variances for averages calculated by integrating over the volume of the ball would be larger by a factor of order $V / V_{0}$ (assuming that the radius of the ball is at least of the order of the correlation length in the medium). An observer living within the ball would identify the spatial averages of the field and of its time derivative with $\bar{\phi}\left(\tau_{0}\right)$ and $\dot{\bar{\phi}}\left(\tau_{0}\right)$, respectively. The point is that these random variables are fluctuating in a predictable manner.

In quantum theory $\bar{\phi}\left(\tau_{0}\right)$ and $\dot{\bar{\phi}}\left(\tau_{0}\right)$ can be sampled from the probability distribution characterized by the mean

$$
\begin{aligned}
& E\left[\bar{\phi}_{i}\left(\tau_{0}\right)\right]=\delta_{i 0} H / \chi_{T}, \\
& E\left[\dot{\bar{\phi}}_{i}\left(\tau_{0}\right)\right]=0,
\end{aligned}
$$

where $\chi_{T}$ is the mass gap (squared) in equilibrium at temperature $T$, by the variances

$$
\begin{aligned}
\operatorname{Var}\left[\bar{\phi}_{i}\left(\tau_{0}\right)\right] & =\frac{1}{V_{0}^{2}} \int_{V_{0}} d^{3} x d^{3} x^{\prime}\left\langle\phi_{i}(x) \phi_{i}\left(x^{\prime}\right)\right\rangle \\
\operatorname{Var}\left[\dot{\bar{\phi}}_{i}\left(\tau_{0}\right)\right] & =\frac{1}{V_{0}^{2}} \int_{V_{0}} d^{3} x d^{3} x^{\prime}\left\langle\dot{\phi}_{i}(x) \dot{\phi}_{i}\left(x^{\prime}\right)\right\rangle
\end{aligned}
$$

and by the covariance

$$
\operatorname{Cov}\left[\bar{\phi}_{i}\left(\tau_{0}\right), \dot{\bar{\phi}}\left(\tau_{0}\right)\right]=\frac{1}{V_{0}^{2}} \int_{V_{0}} d^{3} x d^{3} x^{\prime}\left\langle\frac{1}{2}\left[\phi_{i}(x) \dot{\phi}_{i}\left(x^{\prime}\right)+\dot{\phi}_{i}\left(x^{\prime}\right) \phi_{i}(x)\right]\right\rangle
$$

In the Hartree approximation the interacting system is replaced by an ensemble of free excitations, with mass $\sqrt{\chi_{T}}$. It is not difficult to convince oneself that in this approximation and in the thermal canonical ensemble with density matrix $\propto \exp \left(-H_{0} / T\right)$, where $H_{0}$ is the free Hamiltonian, the probability distribution

\footnotetext{
${ }^{6}$ The probability distribution of the measured values of an observable $\mathcal{O}$ is determined by the characteristic function $\left\langle e^{i k \mathcal{O}}\right\rangle$.
} 
we are interested in is Gaussian. Furthermore the covariance vanishes. Hence the parameters given by (22)-(25) determine the distribution, actually the analogue of the Wigner function, exactly.

The variances (24)-(25) can be estimated analytically, when the radius $R$ of the ball is much larger than the correlation length $\lambda=1 / \sqrt{\chi_{T}}$. It is sufficient to calculate the variances for the quasi-infinite volume $V$ and to multiply the result by $V / V_{0}$, since the $V / V_{0}$ small cells fluctuate independently. One gets

$$
\begin{aligned}
\operatorname{Var}\left[\bar{\phi}_{i}\left(\tau_{0}\right)\right] & =\frac{3 \operatorname{coth}\left(\sqrt{\chi_{T}} / 2 T\right)}{8 \pi \sqrt{\chi_{T}} R^{3}} \\
\operatorname{Var}\left[\dot{\bar{\phi}}_{i}\left(\tau_{0}\right)\right] & =\frac{3 \sqrt{\chi_{T}} \operatorname{coth}\left(\sqrt{\chi_{T}} / 2 T\right)}{8 \pi R^{3}}
\end{aligned}
$$

The dispersion of $\bar{\phi}_{i}$ calculated exactly is smaller by a factor of 2 (1.5) for $R=\lambda(R=2 \lambda)$ and $T=200$ to $400 \mathrm{MeV}$. The dispersion of $\dot{\bar{\phi}}_{i}$ obtained from (28) is off by $20 \%$ (8\%), respectively. For $R<\lambda$ the disrepancy between the analytical formulae and the exact results increases rapidly. Thus, as expected, the fluctuations within the ball depend rather weakly on the environement provided $R \gtrsim \lambda$.

The formalism of ref. [8], reviewed in sect. 2, together with the sampling method proposed in this section enable one to estimate the likelihood of a coherent amplification of the pion field. More precisely, one can calculate the probability that the amplification factor $A_{s}$ given by eq. (21) takes a given value. In such a calculation the size of the initial ball, viz. $R$, appears as a free parameter. Remember, however, that one has to set $\tau_{0}=R$ and that the friction force responsible for the quench is proportional to $1 / \tau$. Thus the likelihood of DCC formation decreases with increasing $R$ : this parameter should be assigned the smallest possible value in order to get the estimate we are looking for.

At this point of the discussion let us remark that the theory we wish to use makes only sense for $R \gtrsim \lambda$. Indeed, the concept of local thermal equilibrium is meaningfull when applied to a cell whose degrees of freedom fluctuate more or less independently from what happens in the neighbor cells. Also, the validity of the Hartree approximation requires the size of the cell to be larger than the Compton wavelength of an excitation. With these arguments in mind we focus on the values of $R$ in the range of one to two correlation lengths ฤ.

\footnotetext{
${ }^{7}$ We cannot exclude that a DCC instability develops in a smaller cell, but we have no theory to deal with such a scenario.
} 


\section{Results and discussion}

We show in Fig. 1 the histograms of the probability $P(A)$ that the amplification factor of the $s=0^{+}$mode is $A_{0} \equiv A$. It was convenient to assign to the parameters of the model the values already used in ref. [8][ Proceeding in this way we could check our results against theirs. The amplification is calculated at $\tau=\tau_{f}=10 \mathrm{fm}$, where the system is in the stationary regime. It is seen from the figure that $P(A)$ falls rapidly with $A$. Clearly, large amplification occurs in a small fraction of events only, especially with the choice $R=2 \lambda$. This is qualitatively similar to the analytical result obtained in ref. [3] using a $1+1$ dimensional toy model, except that in the present case $P(A)$ has a power falling tail at large $A$ :

The histogram corresponding to $T=200 \mathrm{MeV}$ and $R=\lambda$ is fairly well represented by

$$
P(A)=\frac{0.805}{(1+0.27 A)^{3.21}}
$$

The corresponding fit for $T=400 \mathrm{MeV}$ is

$$
P(A)=\frac{0.213}{(1+0.032 A)^{6.959}}
$$

It is impossible to find a good fit reproducing the tail of the histogram with an exponential function, e.g. with $P(A) \propto \exp \left(-a A^{b}\right)$.

It is interesting to inquire what characterizes the initial states leading to large amplifications. It appears that one condition is the smallness of the absolute strength of the initial classical isovector current (calculated using $\bar{\phi}\left(\tau_{0}\right)$ and $\left.\dot{\bar{\phi}}\left(\tau_{0}\right)\right)$. This is ilustrated in Fig. 2, where we show the square of the isovector (resp. isoaxialvector) current versus the amplification factor $A$.

In order to judge what amplification should be considered as "large" one has to estimate the multiplicity of produced pions. This can be done with the help of the formula (cf 15]) :

$$
E \frac{d n}{d^{3} p}=\int d^{4} x \sqrt{-g} \delta\left(\tau-\tau_{f}\right) f(x, p) p^{\mu} u_{\mu}
$$

where $f(x, p)$ is the invariant phase-space density and $u^{\mu}=x^{\mu} / \tau$ is a unit 4vector orthogonal to the hypersurface $\tau=\tau_{f}$, where the particles are countedf? Eq. (31) becomes identical to the eq. (B2) used for the same purpose in ref.

\footnotetext{
${ }^{8}$ Hence, $\Lambda=800 \mathrm{MeV}, \lambda_{r}=7.3, f_{\pi}=92.5 \mathrm{MeV}$. We find the correlation length $\lambda=1.17$ $\mathrm{fm}(0.68 \mathrm{fm})$ at $T=200 \mathrm{Mev}(400 \mathrm{MeV})$.

${ }^{9}$ The textbook formula for the intensity of the black-body radiation is obtained replacing in (31) the constraint $\tau=\tau_{f}$ by $t=$ const, so that $p^{\mu} u_{\mu}=E$, and by setting $f(x, p)=$ $2(2 \pi)^{-3}[\exp (E / T)-1]^{-1}$. The factor 2 is the number of photon polarization states. This example helps fixing the normalizations.
} 
(a)

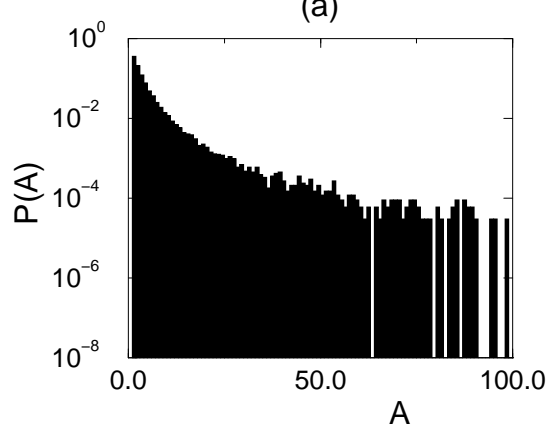

(c)

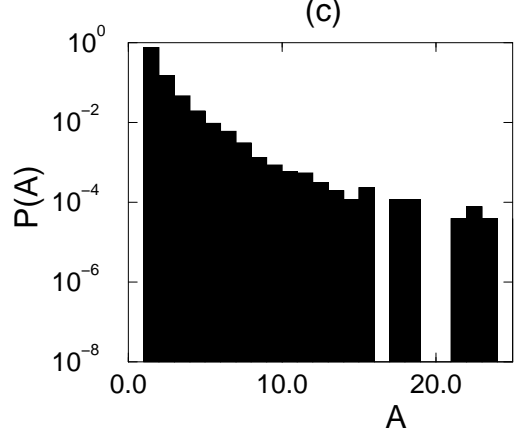

(b)

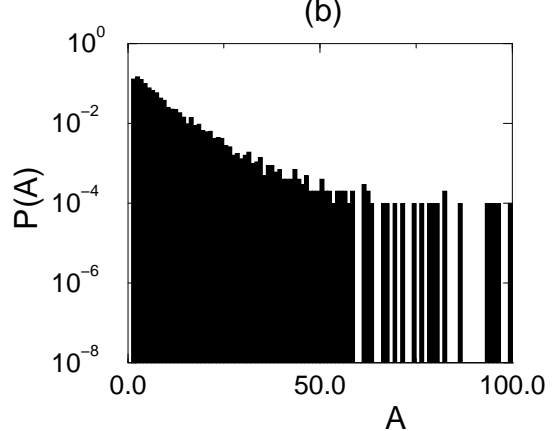

(d)

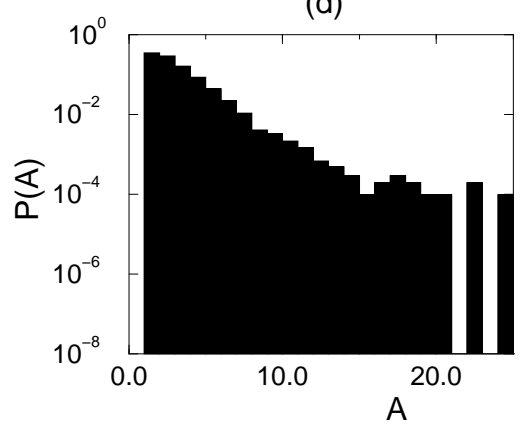

Figure 1: The amplification factor $A$ of the softest mode for (a) $T=200 \mathrm{MeV}$ and $R=\lambda\left(3.2 \times 10^{4} \mathrm{MC}\right.$ events $),(\mathrm{b}) T=400 \mathrm{MeV}$ and $R=\lambda\left(10^{4} \mathrm{MC}\right.$ events $)$, (c) $T=200 \mathrm{MeV}$ and $R=2 \lambda\left(2.5 \times 10^{4} \mathrm{MC}\right.$ events $),(\mathrm{d}) T=400 \mathrm{MeV}$ and $R=2 \lambda$ $\left(10^{4} \mathrm{MC}\right.$ events $)$.

[8], when one goes over to the hyperbolic coordinates (6) and integrates out the delta function 10 . We further set

$$
f(x, p)=N(2 \pi)^{-3} n_{s}^{(b)}
$$

and, as in [8], we relate $s$ to the 4 -momentum $p^{\mu}$ by the obvious relation

$$
p^{\mu} u_{\mu}=\sqrt{\left(s / \tau_{f}\right)^{2}+m_{\pi}^{2}}
$$

The integrand in (31) depends on a single external 4-vector, viz. $p^{\mu}$, and

\footnotetext{
${ }^{10}$ Except for a factor $2 \pi$, coming from the integration over the azimuthal angle, which is missing in (B2) due to a typo.
} 

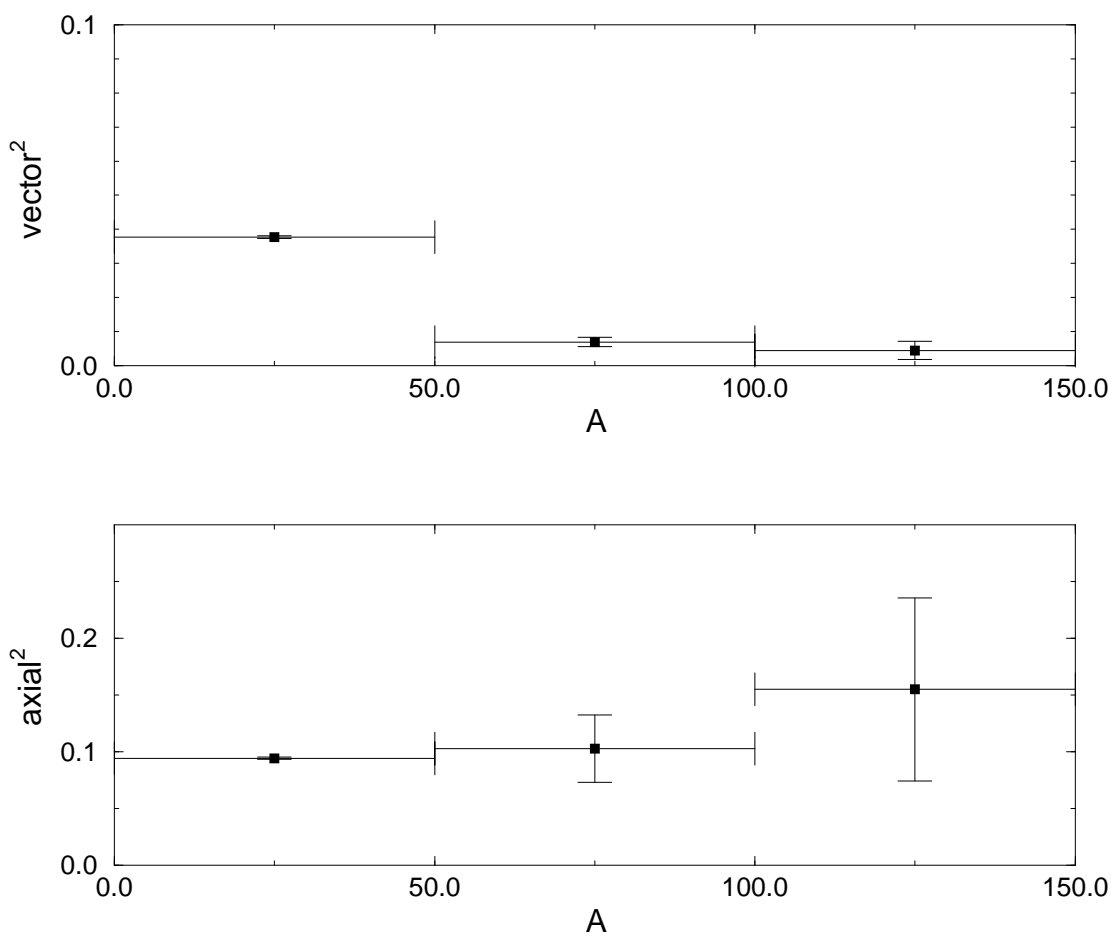

Figure 2: $\mathbf{V}_{\mu} \cdot \mathbf{V}^{\mu}$ [resp. $\mathbf{A}_{\mu} \cdot \mathbf{A}^{\mu}$ ], the square of the initial classical isovector [resp. isoaxialvector ] current (in $\mathrm{fm}^{-6}$ ) versus the amplification factor $A$. We use Monte Carlo data corresponding to $R=\lambda$ and $T=200 \mathrm{MeV}\left(3.2 \times 10^{4} \mathrm{MC}\right.$ events $)$.

therefore the invariant spectrum is flat $\square$ :

$$
E \frac{d n}{d^{3} p}=c A
$$

The constant $c$ on the rhs is varying from one Monte Carlo event to another, but for $A>30$ its average is roughly $c=5 \mathrm{GeV}^{-2}$ for $R=\lambda$ and both $T=200 \mathrm{MeV}$ and $T=400 \mathrm{MeV}$. The flatness of the spectrum is, of course, an artifact of the unrealistic assumption that the boost invariant expansion continues forever. It is worth mentioning that the rhs of (34) does not depend on the choice of $\tau_{f}$, provided the input $f(x, p)$ in (31) is a time independent function of $s$.

\footnotetext{
${ }^{11}$ We are puzzled by the falling DCC spectrum shown in fig. 7 of ref. [8].
} 
At this point one should distinguish between the intrisic DCC dynamics and the extrinsic aspects of DCC formation, those determined by the behavior of the environement of the DCC bubble. A discussion of the latter, which would require the modeling of the collision proces as a whole, is beyond the scope of this paper. Let us only remark that in a real collision process the expansion would last a finite time. The resulting spectrum would be cut, the value of the cut-off reflecting the behavior of the environement. Unfortunately, the predicted total multiplicity depends strongly on this cut-off and cannot be estimated in a reliable manner. However, the rhs of (34) is presumably a reasonable estimate of the invariant momentum space density of soft pion radiation.

A simple example is instructive: Denoting by $h$ the height of the rapidity "plateau", one can parametrize the one-particle spectrum in the central rapidity region writing $E \frac{d n}{d^{3} p}=\frac{h}{\pi\left\langle p_{t}^{2}\right\rangle} \exp \left(-\frac{p_{t}^{2}}{\left\langle p_{t}^{2}\right\rangle}\right)$. In a central $\mathrm{Pb}-\mathrm{Pb}$ colision at the CERN SPS one observes [16] about $200 \pi^{-}$per unit rapidity, i.e. for all pions $h \approx 600$. Thus the invariant momentum space density at very small transverse momentum is roughly $1900 \mathrm{GeV}^{-2}$, where we have used $\left\langle p_{t}^{2}\right\rangle=0.1 \mathrm{GeV}^{2}$. The corresponding density fluctuation is expected to be of the order of $75 \mathrm{GeV}^{-2}$. The rhs of (34) should be significantly larger than the fluctuation, for the DCC signal to be detectable 12 . The signal would be more than three times the fluctuation if $A>45$. Setting $R=\lambda$, the corresponding probability is roughly $4 \times 10^{-3}$. Of course, this is a conditional probability, as it has been assumed that the initial plasma droplet was formed in the collision $\square$.

Summarizing: The mean-field approximation of ref. [8] is combined with a specific sampling method designed to generate the initial values taken by the mean-field and its time derivative. The method rests on the assumption that the medium is initially in a state of local thermal equilibrium. The long-wavelength excitations of the medium are modeled with the linear sigma model. The probability that the amplification of the soft modes has a given magnitude has been estimated. The probability of an observable DCC signal appears small. A crude estimate indicates that in a central $\mathrm{Pb}-\mathrm{Pb}$ collision at CERN SPS this probability is at best of the order of $10^{-3}$. This indication should be taken into account by experimenters designing DCC hunt strategies.

Acknowledgements: We acknowledge useful conversations/correspondence with F. Cooper, B. Jancovici, J. Jurkiewicz, M.A. Lampert, A.H. Mueller, J.Y. Ollitraut, R. Omnes and J. Randrup.

\footnotetext{
${ }^{12}$ Hopefully, a DCC signal can be distinguished from a large fluctuation due to its specific charge structure.

${ }^{13} \mathrm{We}$ get almost the same estimate at 200 and $400 \mathrm{MeV}$. Although at $200 \mathrm{MeV}$ the initial fluctuations are smaller, the friction is weaker, since we take a larger initial ball.
} 


\section{References}

[1] A.A. Anselm, Phys. Lett B217 (1988) 169; A.A. Anselm and M.G. Ryskin, Phys. Lett. B266 (1991) 482.

[2] J.D. Bjorken, Int. J. Mod. Phys. A7 (1992) 4189; Acta Phys. Polon. B23 (1992) 561.

[3] J.P. Blaizot and A. Krzywicki, Phys. Rev. D46 (1992) 246; Phys. Rev. D50 (1994) 442.

[4] K. Rajagopal and F. Wilczek, Nucl. Phys. B399 (1993) 395; Nucl. Phys. B404 (1993) 577.

[5] K. Rajagopal, in Quark-Gluon Plasma 2, ed. R. Hwa, World Scientific, 1995.

[6] J.P. Blaizot and A. Krzywicki, Acta Phys. Polon. 27 (1996) 1687.

[7] F. Cooper, Y. Kluger, E. Mottola and J.P. Paz, Phys. Rev. D51 (1995) 2377.

[8] M.A. Lampert, J.F. Dawson and F. Cooper, Phys. Rev. D54 (1996) 2213; M.A. Lampert, Thesis (University of New Hampshire, December 1996).

[9] J. Randrup, Phys. Rev. Lett. 77 (1996) 1226.

[10] G. Amelino-Camelia, J.D. Bjorken, S.E. Larsson, Phys. Rev. D56 (1997) 6942 .

[11] N.D. Birrell and P.C.W. Davies, Quantum Fields in Curved Space, (Cambridge University Press, Cambridge, 1982).

[12] J. Baacke, K. Heitmann and C. Paetzold, Phys. Rev D57 (1998) 6398; J. Lindig, Not all adiabatic vacua are physical states, hep-th/9808133.

[13] M. Combescure, J. Math. Phys. 33, 3870 (1992).

[14] J. Randrup, private communication.

[15] F. Cooper and G. Frye, Phys. Rev. D10 (1974) 186.

[16] NA49 Collaboration, Quark Matter '97, Tsukuba, Japan, Dec. 1997. 